\title{
Model Parameters Estimation for Image Motion Compensation
}

\author{
Zhong Xunyu, Li Xiaoshan \\ Department of Automation \\ Xiamen University \\ Xiamen, China \\ zhongxunyu@xmu.edu.cn
}

\author{
Zhao Yonggang \\ Tianjin Navigation Instruments Research Institute \\ China Shipbuilding Industry Corporation \\ Jiujiang, China \\ zhaoyonggangcsic@163.com
}

\begin{abstract}
Fast and optimal motion estimation method is proposed for electronic image stabilization. First, an approach for macro-block judgment is presented. Before motion vectors calculation, gradient information is analyzed, only useful reference blocks that are indispensable for accurate motion estimation are selected, by which the number of macro-blocks for subsequent calculation is reduced. Second, in the block matching, an improved SSDA is used to reduce computing cost. Finally, the affine transformation model and similarity transformation model of image motion are created and using least squares method for solving the optimal estimation of model parameters. Experimental results show the accuracy and fast computing speed of the proposed method.
\end{abstract}

Keywords- electronic image stabilization; motion estimation; model parameters; fast matching

\section{INTRODUCTION}

Electronic image stabilization is simpler, flexible, and has advantages in terms of intelligence, size and power consumption than conventional optical image stabilization and mechanical image stabilization; it has been widely used in various mobile camera systems. The block matching method, projection method, feature point method and frequency domain estimation method are used for motion estimation of image sequence [1-4]. Commonly used blockmatching method is to divide the image into several rectangular macro-blocks, solving their motion vectors and then used to estimate the global motion of the image. In order to achieve fast and robust motion estimation of image sequences, the key is to distinguish the useful motion vectors and incorrect motion vectors, and minimize the amount of calculation. With a single two-dimensional rigid body motion model to estimate the motion of image sequences, significant motion estimation errors will occur when view depth changes largely[5]. So, the proposed 2.5-dimensional motion model is better than the two-dimensional affine model [6], and the method of motion vectors segmentation was used in [7]. However, in these methods, the removal of the incorrect macro blocks are all behind the calculation of motion vectors, a considerable portion of time spent in the calculation of these inaccurate motion vectors is bound to have great calculate waste.

In order to reduce the calculate cost and improve the robustness of motion estimation[8,9], in this paper, affine transformation model and the similarity transformation model are used, and combined with the Sequential Similarity
Detection Algorithm (SSDA) and Least Squares Method, an improved fast and optimal motion estimation method is proposed for image motion estimation.

\section{PROCESS OF ELECTRONIC IMAGE STABILIZATION}

The proposed motion estimation for electronic image stabilization consists of two stages. The first stage is preselection of local macro-blocks and macro-blocks matching, and then get all the motion vectors of these selected partial macro-blocks; the second stage is to establish the affine image motion model, and making optimal estimation of model parameters.

Fig. 1 shows the outline of electronic image stabilization, where the proposed motion estimation operation consists of two stages. The first stage is pre-selection of local macroblocks and macro-blocks matching, and then get all the motion vectors of these selected partial macro-blocks; the second stage is to establish the affine image motion model, and making optimal estimation of model parameters.In following sections, we will explain this two-stage operation.

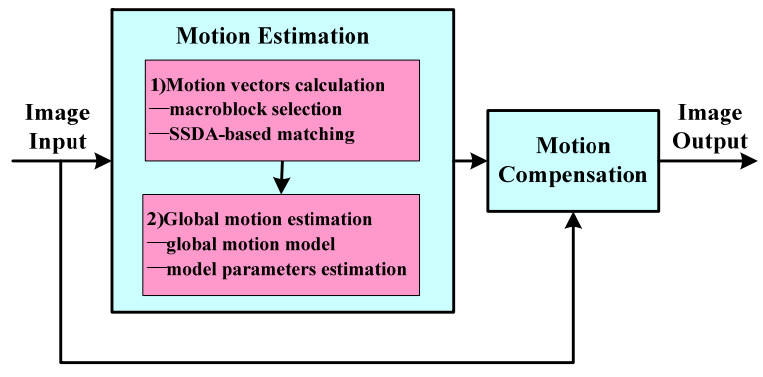

Figure 1. Outline of electronic image stabilization

\section{MACRO-BLOCKS SELECTION AND MARCHING}

First, a whole template region is set in the current sequential image (frame $k$ ). The template is a size slightly smaller than whole image region, then the template area is divided into a number of macro-blocks, and we can select the useful part of their macro-blocks and then calculate their motion vectors.

\section{A. Selection of Local Macro-blocks}

The best matching position of each macro-block is calculated within each local searching area, so we can get motion vector of each macro-block relative to the reference 
image. In order to remove the macro-blocks that may lead to inaccurate motion, we expanded the macro-block selection method [9] as follows.

Step1: Divide the large whole template into smaller local macro-block $\mathrm{C}_{i}(i=0, \ldots, n)$.

Step2: To every macro-block $C_{i}$, set its surrounding macro-blocks as $R_{i}^{1} \sim R_{i}^{4}$ ( Correspond to up, down, left, and right position), calculate the $\mathrm{SAD}$ between $R_{i}^{1} \sim R_{i}^{4}$ and $\mathrm{C}_{i}$ as follow.

$$
\begin{aligned}
D_{i}^{1} & =\sum_{m=1}^{M} \sum_{n=1}^{N}\left|f_{k}\left(x_{i}+m, y_{i}+n\right)-f_{k}\left(x_{i}+m, y_{i}-N+n\right)\right| \\
D_{i}^{2} & =\sum_{m=1}^{M} \sum_{n=1}^{N}\left|f_{k}\left(x_{i}+m, y_{i}+n\right)-f_{k}\left(x_{i}+m, y_{i}+N+n\right)\right| \\
D_{i}^{3} & =\sum_{m=1}^{M} \sum_{n=1}^{N}\left|f_{k}\left(x_{i}+m, y_{i}+n\right)-f_{k}\left(x_{i}-M+m, y_{i}+n\right)\right| \\
D_{i}^{4} & =\sum_{m=1}^{M} \sum_{n=1}^{N}\left|f_{k}\left(x_{i}+m, y_{i}+n\right)-f_{k}\left(x_{i}+M+m, y_{i}+n\right)\right|
\end{aligned}
$$

Where, $\left(x_{\mathrm{i}}, y_{\mathrm{i}}\right)$ is the position coordinates of $C_{i}, f_{k}(x, y)$ is the gray value of pixel $(x, y)$ in the current frame $\mathrm{k}, \mathrm{M} \times \mathrm{N}$ is the size of macro-block.

Step 3: If min $\left(D_{i}^{1}, D_{i}^{2}, D_{i}^{3}, D_{i}^{4}\right)>T_{h}$, then select $C_{i}$, else remove $C_{i}$.

Step 4: If $\mathrm{N}_{\min } \leq n_{\text {sel }} \leq \mathrm{N}_{\max }$, then repeat Step2 and Step3 with adjusted $T_{h}$, else end. Where, $n_{\text {sel }}$ is the number of selected macro-blocks, and $\mathrm{N}_{\min }, \mathrm{N}_{\max }$ are threshold of $n_{\text {sel }}$.

\section{B. SSDA-based Macro-block Matching}

The best matching position of macro-block $C_{i}$ is calculated within its local searching area in reference image (frame $k-1$ ), so we can get motion vector of each selected macro-block, i.e. the measured position coordinates $\left(x_{i}, y_{i}\right)$ of $C_{i}$. Here, we use SAD as similarity measure criterion:

$$
S A D(x, y)=\sum_{i=1}^{M} \sum_{j=1}^{N}\left|f_{k}(x, y)-f_{k-1}(x+i, y+j)\right|
$$

If at a certain point $\left(x^{*}, y^{*}\right)$, the $\operatorname{SAD}\left(x^{*}, y^{*}\right)$ is minimum, then this point is the optimal match position, i.e. the measured position.

In order to reduce computing cost of block matching, we propose an improved SSDA as follow.

For the macro-block $C_{i}$, firstly, in the reference image choose original matched pixel point $\left(x_{0}, y_{0}\right)$ without offset, calculate $\operatorname{SAD}\left(x_{0}, y_{0}\right)$ as initial threshold $T_{0}$, then continual make matching at next pixel points. If $\operatorname{SAD}\left(x_{k}, y_{k}\right)$ exceeds $T_{k-1}$ when during the matching at point $\left(x_{k}, y_{k}\right)$, then stop the matching calculation at once, and set $T_{k}=T_{k-1}$, turn to next point. Otherwise, if $\operatorname{SAD}\left(x_{k}, y_{k}\right)$ Less than $T_{k-1}$, then Update the threshold, set $T_{k}=\operatorname{SAD}\left(x_{k}, y_{k}\right)$. i.e.

$$
T_{k}=\left\{\begin{array}{lc}
S A D\left(x_{k}, y_{k}\right) & \left(T_{k-1}>\operatorname{SAD}\left(x_{k}, y_{k}\right)\right) \\
T_{k-1} & \left(T_{k-1} \leq \operatorname{SAD}\left(x_{k}, y_{k}\right)\right)
\end{array}\right.
$$

The update of optimal match position $\left(x^{*}, y^{*}\right)$ is similar, i.e. $\left(x^{*}, y^{*}\right)=\left(x_{k}, y_{k}\right)$ when $T_{k-1}>\operatorname{SAD}\left(x_{k}, y_{k}\right)$, otherwise, $\left(x^{*}, y^{*}\right)$ keep the former values if $T_{k-1} \leq \operatorname{SAD}\left(x_{k}, y_{k}\right)$.

\section{GLobal Motion Estimation of WhOLE IMAGE}

\section{A. The Model of Image Motion}

Using affine transformations model which could describe panning, rotation, small zooming and deformation, the process can be expressed as:

$$
\left[\begin{array}{l}
x_{i} \\
y_{i}
\end{array}\right]=\left[\begin{array}{ll}
a_{11} & a_{12} \\
a_{21} & a_{22}
\end{array}\right]\left[\begin{array}{c}
X_{i} \\
Y_{i}
\end{array}\right]+\left[\begin{array}{c}
\Delta x \\
\Delta y
\end{array}\right]
$$

Where, $\left(X_{\mathrm{i}}, Y_{\mathrm{i}}\right)\left(i=1,2, \ldots, n_{\mathrm{sel}}\right)$ is position of the $i$-th selected macro-block $C_{\mathrm{i}},\left(X_{\mathrm{i}}, Y_{\mathrm{i}}\right)$ is registered in current frame $k$ (called as Registered Position); $\left(x_{\mathrm{i}}, y_{\mathrm{i}}\right)$ is the matchingobtained position of $C_{\mathrm{i}}$ in reference frame (called as Measured Position); $a_{11}, a_{12}, a_{21}, a_{22}$ are rotation parameters, including small zooming and deformation; $\Delta \mathrm{x}, \Delta y$ are the offset of image panning in $\mathrm{X}$-axis and $\mathrm{Y}$-axis respectively.

\section{B. Estimation of Motion Model Parameters}

Show as Fig. 2, registered position $\left(X_{\mathrm{i}}, Y_{\mathrm{i}}\right)$ of each macroblock is transformed to estimated position $\left(\hat{x}_{i}, \hat{y}_{i}\right)$ using (3). Minimizing errors between estimated positions and measured ones leads to optimum parameters by Least Squares Method.

Estimated parameters $\left(\hat{a}_{11}, \hat{a}_{12}, \hat{a}_{21}, \hat{a}_{22}, \Delta \hat{x}, \Delta \hat{y}\right)$ are determined to minimize the functions $E_{\mathrm{m}}$.

$$
E_{m}=\sum_{i=1}^{n}\left(\left(\hat{x}_{i}-x_{i}\right)^{2}+\left(\hat{y}_{i}-y_{i}\right)^{2}\right)
$$

Where, $\left(\hat{x}_{i}, \hat{y}_{i}\right)$ is the estimated position of $i$-th macroblock calculated with estimated model parameters.

Based on affine transformations model (3), the process of parameters estimation designed as follow.

Step1: Calculating the Measured Position $\left(x_{i}, y_{i}\right)(i=1,2, \ldots$, $\left.n_{\mathrm{sel}}\right)$ of each selected macro-block $C_{\mathrm{i}}\left(X_{i}, Y_{i}\right)$ by SSDA-based block matching method.

Step2: Building the known design matrix $\boldsymbol{A}: \boldsymbol{A}=\left[\mathrm{X}_{1} \mathrm{Y}_{1} 0\right.$ $010 ; \mathrm{X}_{2} \mathrm{Y}_{2} 0010 ; \ldots ; \mathrm{Xn}_{\text {sel }} \mathrm{Yn}_{\text {sel }} 0010 ; 00 \mathrm{X}_{1} \mathrm{Y}_{1} 01$; 0 $0 \mathrm{X}_{2} \mathrm{Y}_{2} 01 ; \ldots ; 00 \mathrm{Xn}_{\text {sel }} \mathrm{Yn}_{\text {sel }} 01$ 1].

Step3: Building the known vector $\boldsymbol{B}: \mathrm{B}=\left[\begin{array}{llllll}x_{1} & x_{2} & \ldots & x_{\text {nsel }} & y_{1}\end{array}\right.$ $\left.y_{2} \ldots y_{\text {nsel }}\right]^{\mathrm{T}}$.

Step4: Building the unknown model parameter matrix $\boldsymbol{H}_{\text {Affine }}: \boldsymbol{H}_{\text {Affine }}=\left[\begin{array}{llllll}a_{11} & a_{12} & a_{21} & a_{22} & \Delta x & \Delta y\end{array}\right]^{\mathrm{T}}$.

Step5: Corresponds to the affine transformation (3), create the image motion model: $\boldsymbol{B}=\boldsymbol{A} \boldsymbol{H}_{\text {Affine }}$.

Step6: Calculate linear unbiased estimation of the parameters matrix $\boldsymbol{H}_{\text {Affine }}$ by Least Squares Method:

$$
\hat{\boldsymbol{H}}_{\text {Affine }}=\left(\boldsymbol{A}^{\mathrm{T}} \boldsymbol{A}\right)^{-1} \boldsymbol{A}^{\mathrm{T}} \boldsymbol{B}
$$




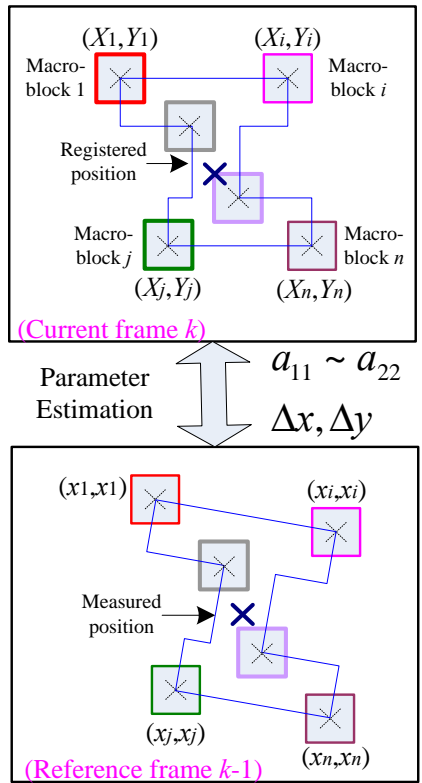

Figure 2. Model parameters estimation

\section{Calculation of Global Image Motion}

Due to the six parameters of $\boldsymbol{H}_{\text {Affine }}$ can not well represent the rotation and zooming of image motion, so we should transform the affine model into similarity transformation model which defined as follow.

$$
\left[\begin{array}{l}
x_{i} \\
y_{i}
\end{array}\right]=K\left[\begin{array}{cc}
\cos \theta & -\sin \theta \\
\sin \theta & \cos \theta
\end{array}\right]\left[\begin{array}{c}
X_{i} \\
Y_{i}
\end{array}\right]+\left[\begin{array}{c}
\Delta x \\
\Delta y
\end{array}\right]
$$

Where, $K$ is zooming factor, $\theta$ is rotation angle, $\Delta \mathrm{x}, \Delta y$ are panning offsets in the $\mathrm{X}$-axis and $\mathrm{Y}$-axis respectively, $\left(x_{\mathrm{i}}, y_{\mathrm{i}}\right)$ and $\left(X_{\mathrm{i}}, Y_{\mathrm{i}}\right)$ are pixel coordinates in reference frame and current frame respectively.

By comparing the affine model (3) and the similarity transformation model (6), we have $a_{11}=K \cos \theta, a_{12}=-K \sin \theta$, $a_{21}=K \sin \theta, a_{22}=K \cos \theta$. So, calculate the rotation angle as follow:

$$
\hat{\theta}=\frac{1}{4}\left(\begin{array}{c}
\arctan \frac{-\hat{a}_{12}}{\hat{a}_{11}}+\arctan \frac{-\hat{a}_{12}}{\hat{a}_{22}} \\
+\arctan \frac{\hat{a}_{21}}{\hat{a}_{11}}+\arctan \frac{\hat{a}_{21}}{\hat{a}_{22}}
\end{array}\right)
$$

Calculate the zooming factor as follow:

$$
\hat{K}=\frac{1}{4}\left(\frac{\hat{a}_{11}}{\cos \hat{\theta}}+\frac{-\hat{a}_{12}}{\sin \hat{\theta}}+\frac{\hat{a}_{21}}{\sin \hat{\theta}}+\frac{\hat{a}_{22}}{\cos \hat{\theta}}\right)
$$

The panning offsets of $\mathrm{X}$-axis and $\mathrm{Y}$-axis are same as $\Delta \hat{x}, \Delta \hat{y}$. Estimated global motion parameters, i.e. $\hat{\theta}, \hat{K}$, $\Delta \hat{x}$ and $\Delta \hat{y}$, could be filtered for motion smoothing.

\section{EXPERIMENTS}

Fig. 3- Fig. 10 show the experiment results, and the PSNR between Fig. 3 and Fig. 4 is 8.1929, the PSNR between Fig. 3 and Fig. 7 is 12.0384; the PSNR between Fig. 3 and Fig. 5 is 7.2867, the PSNR between Fig. 3 and Fig. 8 is 12.1781, the proposed methods have a good real time and accuracy for global motion estimation.

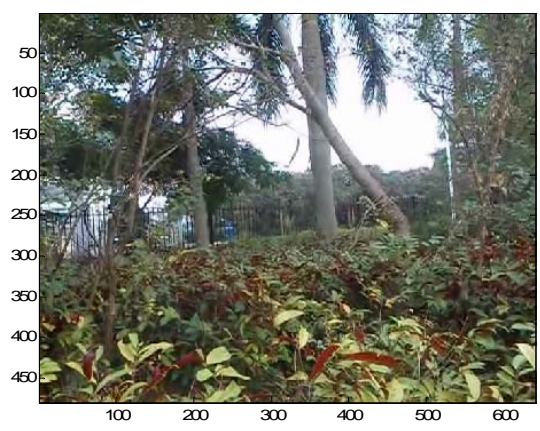

Figure 3. Reference image

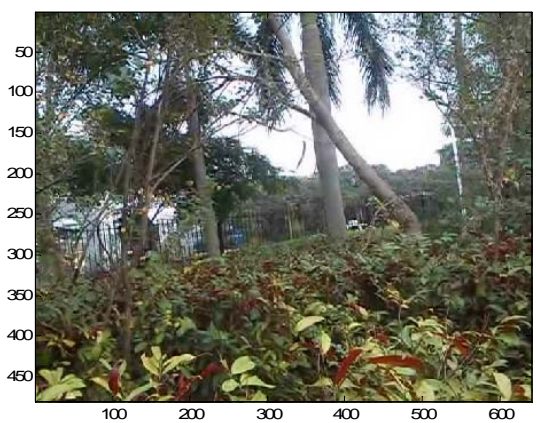

Figure 4. Motive image 1

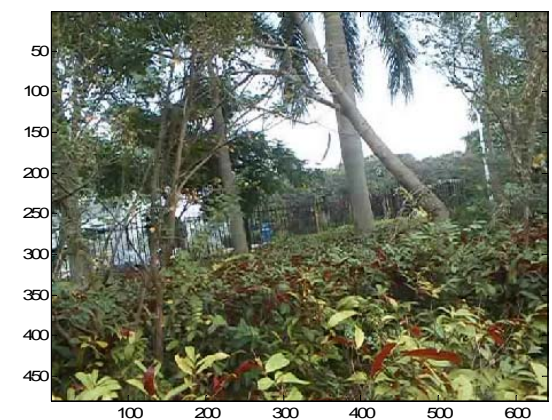

Figure 5. Motive image 2 


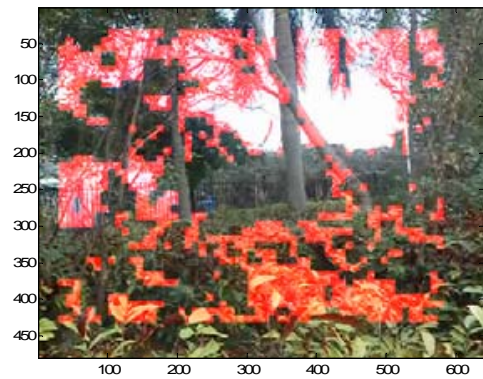

Figure 6. Selected macro-blocks(drown as red regions)

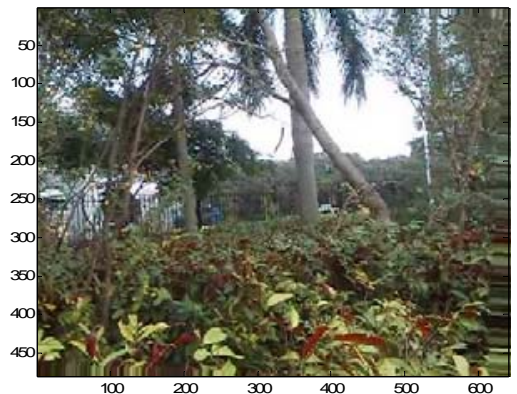

Figure 7. Motion-compensated image of Fig. 4

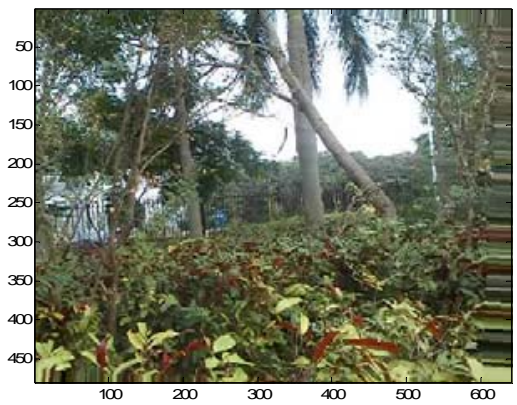

Figure 8. Motion-compensated image of Fig. 5

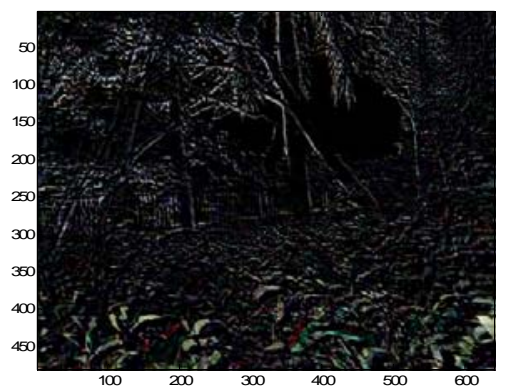

Figure 9. Difference image of Fig. 3 and Fig. 7

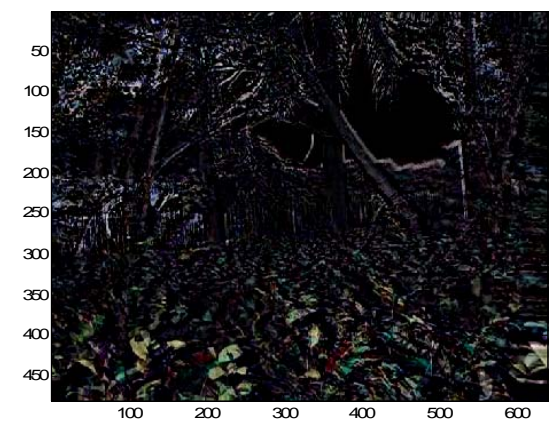

Figure 10. Difference image of Fig. 4 and Fig. 8

\section{REFERENCES}

[1] Uomori K, Morimura A, Ishii H, "Automatic Image Stabilizing System by Full-digital Signal Processing," IEEE Trans on Consumer Electronics, 1990.3, pp510-519.

[2] Sauer K, Schwartz B, "Efficient Block Motion Estimation Using Integral Projections,” IEEE Trans on Circuits and Systems for Video Technology, 1996.5, pp513-518.

[3] Chang JY, Hu WF, Cheng MH, "Digital Image Translational and Rotational Motion Stabilization Using Optical Flow Technique," IEEE Trans on Consumer Electronics, 2002.1, pp108-115.

[4] Erturk S, "Digital Image Stabilization with Sub-image Phase Correlation Based Global Motion Estimation,” IEEE Trans on Consumer Electronics, 2003.4, pp1320-1325.

[5] C. Morimoto, R. Chellappa, "Fast Electronic Digital Image Stabilization," Proc. of 13th IEEE ICPR. Los Alamitos: IEEE Comput. Soc. Press, 1996, pp284-288.

[6] Yang Yudong, Xu Guangyuo, Zhu Zhigang, "Method of 2.5 D Inter Frame Motion Estimation," JOURNAL OF TSINGHUA UNIVERSITY (SCIENCE AND TECHNOLOGY), 1997.9, pp78-81.

[7] J. Heuer, A. Kaup, "Global Motion Estimation in Image Sequences Using Robust Motion Vector Field Segmentation,” Proc. ACM Multimedia 99. New York: ACM, 1999, pp261-264.

[8] H.K. Cheung, W.C. Siu, "Fast Global Motion Estimation for Sprite Generation,” Proceedings of the IEEE International Symposium on Circuits and Systems (ISCAS), Arizona: IEEE, 2002, pp26-29.

[9] H. Okuda, M. Hashimoto, K. Sumi, S. Kaneko, "Optimum Motion Estimation Algorithm for Fast and Robust Digital Image Stabilization," IEEE Transactions on Consumer Electronics, 2006.1,pp276-280. 